\title{
Liquid crystalline polymers: The effects of chain disruptors
}

C. K. S. Pillai

\author{
Regional Research Laboratory, Thiruvananthapuram - 695 019, India.
}

\begin{abstract}
The effect of chain disruptors, both novel and existing, as comonomers on the transition temperatures of liquid crystalline polymers was investigated. Novel comonomers prepared from cost effective natural materials such as cardanol and copolymerised with 4-hydroxy benzoic acid (4-HBA) showed a decrease in transition temperature, but the polyesters started decomposing prior to melting except the bromine containing polyester. Copolyesters of 4-hydroxyphenylacetic acid (4-HyPA) and 4-hydroxyphenylpropionic acid (4-HyPP) with 4-HBA showed fairly good nematic texture with comparatively high thermal stability indicating possibility for further studies. The effects of azophenyl group and di-amide links were also investigated.
\end{abstract}

\section{INTRODUCTION}

LC polymers are a new class of materials having unusual properties (ref.1-2) and represent one of the most exciting developments in high performance materials. They are characterised by the existence of mesophases that exhibit a pronounced anisotropy in shape. This generates organised fluid phases either on melting (thermotropic) or on dissolution (Lyotropic). Making use of the anisotropy in processing of LC polymers, it was possible to achieve impressive properties for the production of high performance (eg. high strength, high modulus, high heat resistance, that are close to theoretical values) fibre, plastics etc. The intractability and insolubility of wholly aromatic homopolyesters and polyamides triggered research world wide to bring down the melting point to a processable range without destroying the mesogenic order. The first successful attempt in this line has been reported by Kuhfuss and Jackson and independently by Roviello and Sirigu. Commercial interest has centered on thermotropic MCLCPs to permit melt processing particularly injection moulding. A number of LC polymers such as Xydar (Dartco), Vectra (CelaneseHoechst, X 7G (Eastman) etc. have been commercialised, but it is reported that they still have processing problems because their transition temperatures are above $300^{\circ} \mathrm{C}$. The need for further studies in this line is indicated. In this paper, the effect of certain chain disruptors used as comonomers on the mesophase properties being studied in this laboratory is discussed.

\section{EXPERIMENTAL}

The polymers reported in this paper were prepared as per procedure reported elsewhere (ref. 1-21). Details of the characterisation, thermal and phase behaviour studies provided in this report appear elsewhere in this conference proceedings.

\section{RESULTS AND DISCUSSION}

Fig 1 gives the structures of a few chain disruptors used in the present work (ref. 1). Some are known and others novel molecules. These novel molecules, except the first two (on which publications are limited), are derived from natural materials so that their cost is comparatively low. It is well known that cost of the monomer molecules of the LC polymers continue to be one of the serious problems faced by the industry. So we tried to prepare comonmers from natural materials such as cardanol (price Rs. 15 per $\mathrm{Kg}$ ).

\section{Studies on novel LCPs containing chain disruptors developed from cost effective natural monomers}

One of the early derivatives of cardanol was the successful synthesis of a comonomer, 8-(3hydroxyphenyl)octanoic acid(HPOA) by phase transfer catalysed permanganate oxidation. The significance of this monomer is its structure having both a molecular 'kink' and a flexible segment. It gave a copolyester with p-hydroxy benzoic acid (pHBA). [Poly(p-hydroxybenzoate-co-hydroxy phenyloctanoate)] was liquid 
crysatlline with nematic texture and gave a transition temperature of $256^{\circ} \mathrm{C}$ (ref. 2-5) (see Fig.3). However, its thermal properties were not encouraging. Another polymer [poly(2-bromo-4hydroxybenzoate-co-3-hydroxyphenyl octanoate)] containing a - $\mathrm{Br}$ group was synthesised. This polymer also showed LC behaviour. Composition variation study carried out on these polymers indicated that the bromoderivative has a good range of nematic phase for processability studies (ref. 6). A number of related systems are under investigation (ref. 7-9).

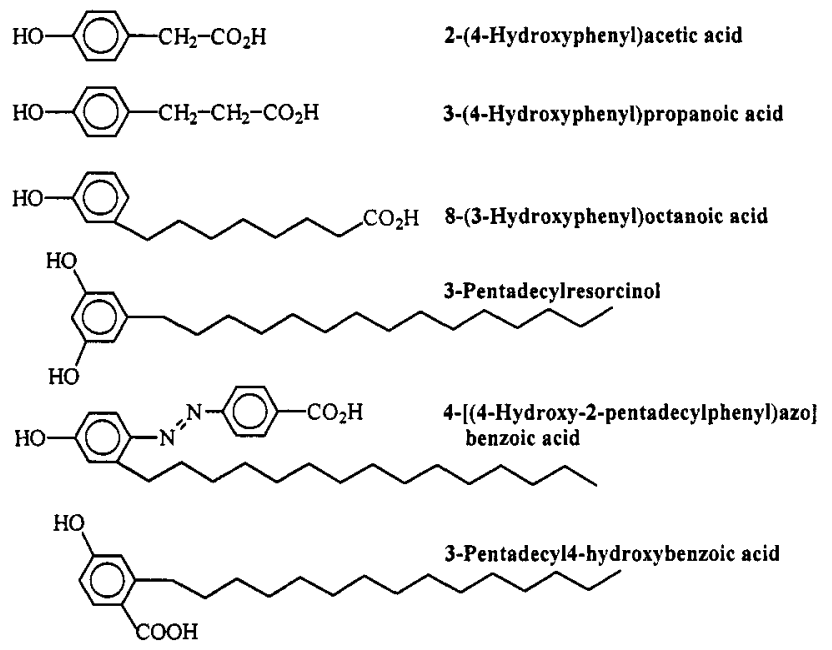

Fig. 1 Chain disruptors under study

A novel LC polymer containing azophenyl group was synthesised by performing a diazotisation reaction between cardanol or pentadecyl phenol and p-aminobenzoic acid and polymerization of the resulting monomer to get poly 4-[4-hydroxy-2-pentadecyl phenyl)azo]benzoic acid. Azo based LC polymers are well known to give non-linear optical behaviour and for such applications the polymers should not absorb in the UV range of $>500 \mathrm{~nm}$. The LC polymer prepared did not absorb in this region indicating possibly that this polymer or related structures might show useful non-linear optical properties. Based on this azophenyl mesogen, a series of main chain and side chain (with mesogen directly attached to the acrylate group) LC polymers were prepared and their properties studied. It was interesting to note that the presence of the mesogen induced the long side chain of pentadecyl phenol to align along the molecular long axis (ref. 913).<smiles>[Y]C(=O)Oc1cccc(CCCCC(=O)Oc2ccc(OC(C)(C)C)cc2)c1</smiles>

Fig. 2. Structure of Poly(p-hydroxybenzoate-co-hydroxy phenyloctanoate).

\section{Studies on the effect of flexible segments in the main chain attached directly to the aromatic ring.}

Both HyAA and HyPP are typical candidate materials for LC formation. Kircheldorf and his group studied the copolymerisation of HyPP with pHBA, but as they got only a low molecular weight polymer which exhibited birefringence, they could not identify whether it is really due to LC formation or not. We have been successful in homopolymerising and copolymerising (with pHBA) these monomers to fairly high molecular weight polyesters that exhibited good nematic textures. The Copolyester of HyAA with pHBA (patents filed) appears to have all properties for further studies in the sense that apart from its showing stable nematic phase, it has a thermal stability of $T_{5}$ of $400^{\circ} \mathrm{C}$ which can be considered good comparing its aliphatic $\mathrm{CH}_{2}$ group attached to its aromatic moiety (ref. 14-15). As the cost the monomers are low when compared to the expensive hydroxy naphthoic aicd monomers, this polymer system has every characteristic for being considered for a serious study. 


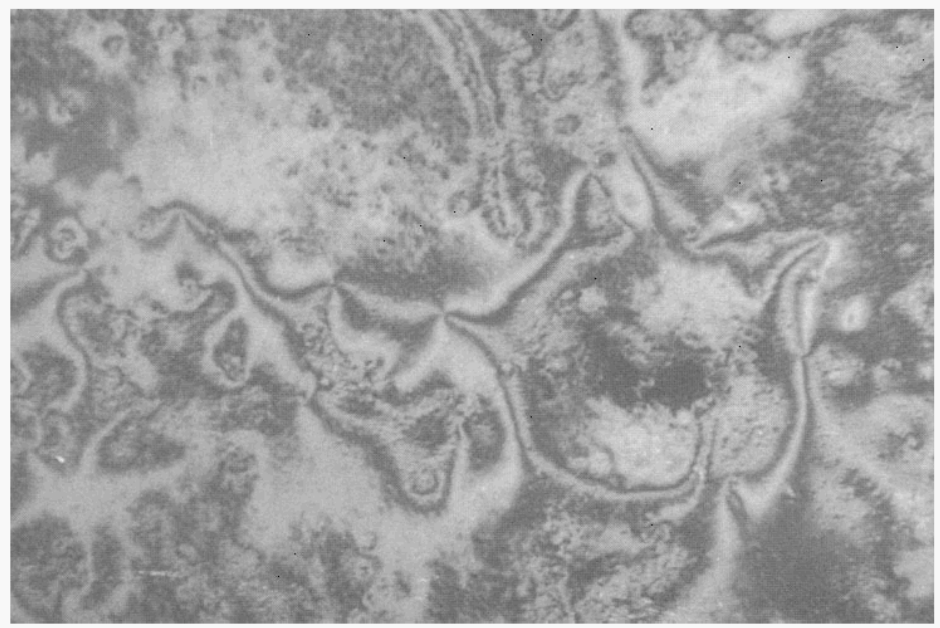

Fig. 3. Schlieren nematic texture of azobenzene based polymers.

\section{Synthesis of Ternary Polvesters:}

Ternary polyesters were also synthesised with Hydroquinone (HQ), Terephthalic acid (TA) and 3-(4Hydroxyphenyl)propionicacid (HyPP) / (4-Hydroxyphenyl )acetic acid (HyPA) and characterised. DSC measurements and PLM studies revealed that the ternary polyesters containing above $80 \mathrm{~mol} \%$ of the hydroxy acids showed nematic LC transition at $243^{\circ} \mathrm{C}$ and $235^{\circ}$ respectively in the case of coployesters of HyPP and HyPA. The ternary polyesters display under the polarising microscope LC phase upto temperatures of $345^{\circ} \mathrm{C}$ where rapid thermal degradation prevented further investigations. The present study showed that by condensing HQ and TA with HyPP / HyPA, as the number of methylene group in the hydroxyacid increases, $\mathrm{Tm}$ decreases and it has reduced upto $235^{\circ} \mathrm{C}$ with $\mathrm{HyPP}$ (ref. 14,15) (for details see the paper appearing elsewhere).

\section{Synthesis and characterisation of Polv(ester amides) containing di-amide links:}

Recently, we reported the synthesis of a poly(ester amide), poly[bis(terephthalate butyramide) hexane, PBTBH (I) having comparatively a large aliphatic segment which normally would not favour the formation of a mesophase. This polymer is prepared through the amido diol route whereby a di-amide link is introduced by the aminolysis of lactone by a diamine to get an amido diol which is later polycondensed with an aromatic dicarboxylic aicd. On heating, it exhibited thermotropic behaviour giving nematic threaded textures typical of LC phase. We ascribed this behaviour to the additional influence of the rigid double bond character of the amide group coupled with the extensive hydrogen bonding of the di-amide link. Further investigations revealed that this polymer also exhibits lyotropic behaviour. Fibres could be drawn by cold drawing (ref. 17-21). It is proposed to study this system in detail.<smiles>CC(C)(C)OCCCC(=O)NCCCCCCNC(=O)CCCOC(=O)c1ccc(C(C)(F)F)cc1</smiles>

In short, we have investigated the effects of molecular kinks and flexible segments on the transition temperatures. In the first system, HPOA, the molecular kink and flexible segment were built in the same molecule and its copolyester with pHBA showed a transition temperature of $256^{\circ} \mathrm{C}$, but composition variation study and thermal stability indication its melt processability is not favourable. But the presence of a bromine atom at the ortho position of hydroxyl group of $\mathrm{pHBA}$ widened the range of melt processability. We also note that the copolyester of HyPA with $\mathrm{pHBA}$ is interesting although one may doubt its stability to oxidation. This copolyester shows a transition temperature at $230^{\circ} \mathrm{C}$ with a perfect nematic texture and a 
thermal stability of $\mathrm{Ti}$ at $400^{\circ} \mathrm{C}$. As there is no work reported on this system, it would be interesting to further investigate this copolyester and its ternary polyesters. Similar observations have been made with the monomer, HyPP as well.

\section{ACKNOWLEDGEMENT}

Thanks are due to Sri V. S. Prasad, Mrs. J.D Sudha, Dr. M. Saminathan, Mrs. Shiney Abraham, Mrs. Veena Vijayanathanan and Mrs. S. Rajalekshmy.

\section{REFERENCES}

1. C.K.S.Pillai, In Handbook of Polymeric Engineering Materials, (Ed) N.P.Cheremisinof, Marcel Dekker, New York (1997), p.411-438.

2. C.K.S.Pillai, D.C.Sherrington and A.Sneddon, Polymer, 33, 3968 (1992).

3. C.K.S.Pillai, D.C.Sherrington and A.Sneddon, Indian Patent, No. 677/Del/92 dtd. 29.7.1992.

4. C.K.S.Pillai, D.C.Sherrington and A.Sneddon, Indian Patent, No. 678/Del/92 dtd. 29.7.1992.

5. C.K.S.Pillai, D.C.Sherrington and A.Sneddon, Indian Patent, No. 679/De1/92 dtd. 29.7.1992.

6. Shiney Abraham, V. S. Prasad and C.K.S.Pillai, Unpublished Results (1997).

7. Veena Vijayanathan, V. S. Prasad and C.K.S.Pillai, Unpublished Results (1997).

8. S.Rajalekshmi and C.K.S.Pillai, Macromolecules: Recent Developments, (Eds) S. Venkatachalam, V.C.Joseph, R.Ramaswamy and V.N.Krishnamurthy, Allied Publishers, Vol. II, pp.591, New Delhi (1995).

9. M.Saminathan, C.K.S.Pillai and C.Pavithran, Macromolecules, 26, 7103 (1993).

10. M.Saminathan and C.K.S.Pillai, Communicated to Macromolecules (1997).

11. M.Saminathan and C.K.S.Pillai, Communicated to J.Polym.Sci., Polym.Chem.Edn.,(1997).

12.M.Saminathan, C.K.S.Pillai and C.Pavithran, Polymer Science: Recent Developments, I.S Bharadwaj (Ed), Allied Publishers, Vol 1, p.458-463, New Delhi (1994).

13. M. Saminathan, Synthesis and Characterisation of Novel Liquid Crysatl Polymers Containing Azobenzene Mesogen, $\mathrm{PhD}$ thesis, Regional Research Laboratory, Thiruvananthapuram and Kerala University, Thiruvananthapuram (1996).

14. V. S.Parsad, M. Saminathan and C. K. S. Pillai, Communicated to Macromolecules, 1997.

15. V. S.Parsad, M. Saminathan and C. K. S. Pillai, A process for the preparation of "poly(hydroxy phenyl acetic acid), patent filed, 1997.

16. V. S.Parsad, M. Saminathan and C. K. S. Pillai, A process for the preparation of poly(hydroxy phenyl acetic acid-co- parahydroxybenzoic acid), patent filed, 1997.

17. J. D. Sudha, C. K. S. Pillai and S. Bera, J. Polym. Mater., 13, 317 (1996).

18. J. D. Sudha, C. K. S. Pillai and S. Bera, Polymer Science: Recent Developments, Ed. I. S. Bharadwaj, Allied Publishers, New Delhi (1994 ), p.424-430.

19. J. D. Sudha and C. K. S. Pillai, Macromolecules: Current Trends (Eds.) S.Venketachalam ,V. C. Joseph, R . Ramaswamy and V. N. Krishnamoorthy, Allied publishers, New Delhi (1995) , P .557

20. J.D.Sudha, S.Bera and C.K.S.Pillai, Polymer Science: Recent Developments, I.S.Bharadwaj (Ed), Allied Publishers, Vol.1, p.424-430, New Delhi (1994).

21. J.D.Sudha and C.K.S.Pillai, In Macromolecules: Recent Developments, (ed.) S. Venkatachalam, V.C.Joseph, R.Ramaswamy and V.N.Krishnamurthy, Allied Publishers, Vol. II, pp.577, New Delhi (1995). 